Discrete Comput Geom 29:239-255 (2003)

DOI: $10.1007 / \mathrm{s} 00454-002-2809-1$

Geometry

\title{
An Isoperimetric Theorem in Plane Geometry*
}

\author{
Alan Siegel \\ Courant Institute of Mathematical Sciences, New York University, \\ New York, NY 10012, USA \\ siegel@cs.nyu.edu
}

\begin{abstract}
Let $P$ be a simple polygon. Let the vertices of $P$ be mapped, according to a counterclockwise traversal of the boundary, into a strictly increasing sequence of real numbers in $[0,2 \pi)$. Let a ray be drawn from each vertex so that the angle formed by the ray and a horizontal line pointing to the right equals, in measure, the number mapped to the vertex. Whenever the rays from two consecutive vertices intersect, let them induce the triangular region with extreme points comprising the vertices and the intersection point. It is shown that there is a fixed $\alpha$ such that if all of the assigned angles are increased by $\alpha$, the triangular regions induced by the redirected rays cover the interior of $P$.

This covering implies the standard isoperimetric inequalities in two dimensions, as well as several new inequalities, and resolves a question posed by Yaglom and Boltanskiľ.
\end{abstract}

\section{Summary}

The isoperimetric theorem states that among all planar regions with a given boundary length $p$, the disk has the maximum area. This bound, which is also known as the isoperimetric inequality, dates back to antiquity. There are also more specialized forms of the inequality for simple polygons. We establish a topological formulation that implies and generalizes the traditional isoperimetric inequalities for the plane.

Unless otherwise stated, all polygons and boundary curves are assumed to be simple and planar. If $P$ is a polygon, let $P^{\text {rgn }}$ denote the bounded polygonal region with $\partial\left(P^{\mathrm{rgn}}\right)=P$. Let $\operatorname{Area}(P)=\operatorname{Area}\left(P^{\mathrm{rgn}}\right)$.

Definition 1. Let $\ell$ be a ray or directed line. The angular direction of $\ell$ is the measure of the angle formed by $\ell$ and a horizontal ray $h$ that originates on $\ell$ and runs to the right. The measure of such an angle is taken in a counterclockwise direction from $h$ to $\ell$.

* This research was supported in part by NSF Grant CCR-9503793. 


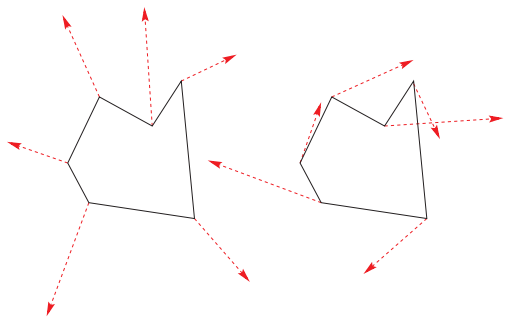

Fig. 1

Definition 2. Let $P$ be an $n$-gon with the consecutive vertices $v_{1}, v_{2}, \ldots, v_{n}$, which are sequenced in counterclockwise order around $P$. Let, for $i=1,2, \ldots, n, r_{i}$ be an infinite ray that originates at vertex $v_{i}$ and has an angular direction $\theta_{i}$. The rays $r_{1}, r_{2}, \ldots, r_{n}$ are called a monotone slicing for $P$ if $\theta_{1}<\theta_{2}<\cdots<$ $\theta_{n}<2 \pi+\theta_{1}$. We say that the slicing rays are associated with $P$, and the angles $\theta_{1}, \ldots, \theta_{n}$ are associated with the rays.

Definition 3. Let $P$ be an $n$-gon and let $r_{1}, r_{2}, \ldots, r_{n}$ be a monotone slicing for $P$. Let $t_{j}$ be the region whose boundary is the triangle defined by the base $\overline{v_{j} v_{j+1}}$ and the intersection of the consecutive ${ }^{1}$ rays $r_{j}$ and $r_{j+1}$ as the opposing vertex, provided $r_{j} \cap r_{j+1}$ exists, and let $t_{j}=\emptyset$ otherwise. We call $t_{j}$ a slicing triangle for $P$, and say that the set of slicing triangles $\left\{t_{j}\right\}_{j=1}^{n}$ is associated with the rays $\left\{r_{i}\right\}_{i=1}^{n}$.

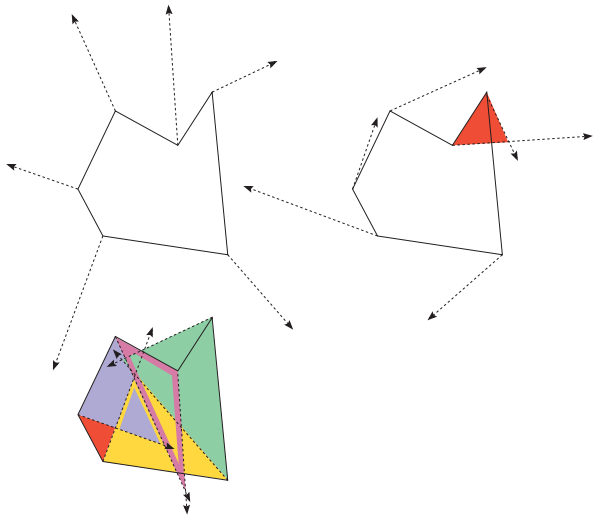

Fig. 2

Theorem 1. Let $P$ be an n-gon with consecutive vertices $v_{1}, v_{2}, \ldots, v_{n}$, and let $r_{1}, r_{2}, \ldots, r_{n}$ be a monotone slicing for $P$ with associated angles $\theta_{1}, \theta_{2}, \ldots, \theta_{n}$. Let $R(\alpha)=\left\{r_{j}(\alpha)\right\}_{j=1}^{n}$ be the monotone slicing for $P$ with associated angles $\theta_{j}+\alpha$, for $j=1,2, \ldots, n$. Let $\left\{t_{j}(\alpha)\right\}_{1}^{n}$ be the set of slicing triangles associated with $R(\alpha)$. Then for some fixed $\alpha$,

$$
P^{\mathrm{rgn}} \subset \bigcup_{j} t_{j}(\alpha)
$$

That Theorem 1 is an isoperimetric inequality can be seen from the following corollaries, which are proved in Section 2.

Corollary 1. Let $F$ be a polygon. Then among all polygons with edges congruent to those of $F$, those polygons whose vertices lie on a circle have the greatest area.

Of course, Corollary 1 is standard. The proof is algorithmic. Let $G$ be a polygon that is inscribed in a circle, and which has sides congruent to those of $F$. The construction will split $G^{\mathrm{rgn}}$ into a collection of $n$ slicing triangles and map them, via individual affine contractions, onto a set of slicing triangles that cover $F^{\mathrm{rgn}}$ even if $F$ is not convex.

\footnotetext{
${ }^{1}$ Of course, the index value $n+1$ is interpreted as 1 .
} 
Definition 4. Let $\varphi(s)$ be a curve parameterized by arclength. Let $\eta(s)$ be the angular direction of a normal to $\varphi$ at $\varphi(s)$. We say that $\varphi$ is piecewise smooth if it can be partitioned into a finite number of pieces where $\eta(s)$ is continuously differentiable on the closure of each piece. Let $\eta(s+)=\lim _{t \downarrow s} \eta(t)$, and let $\eta(s-)=\lim _{t \uparrow s} \eta(t)$.

Corollary 2. Let $C$ be a piecewise smooth simple closed curve with perimeter $p$ and which encloses a region with area $A$. Let $C$ be defined by $\varphi(s)$ with arclength parameter $s$, where $0 \leq s \leq p$. Let $\eta(s)$ be the angular direction of the outward normal to $C$ at $\varphi(s)$. Let $\theta(s)$ be piecewise smooth and strictly increasing with $\theta(p)=2 \pi+\theta(0)$. Let $\dot{\theta}(s)=(d / d s) \theta(s)$. Then

$$
A \leq \max _{\alpha}\left\{\int_{0}^{p} \frac{\cos ^{2}(\alpha+\theta(s)-\eta(s))}{2 \dot{\theta}(s)} d s\right\} .
$$

To see that this is an isoperimetric inequality, let $\theta(s)=2 \pi s / p$. Substituting gives

$$
A \leq \max _{\alpha}\left\{\int_{0}^{p} \frac{p \cos ^{2}(\alpha+\theta(s)-\eta(s))}{4 \pi} d s\right\} \leq \frac{p}{4 \pi} \int_{0}^{p} d s=\frac{p^{2}}{4 \pi},
$$

and equality can hold only if $d \eta(s) / d s$ is constant, which is to say that $C$ is a circle. This special case is the most common formulation of the isoperimetric inequality in two dimensions.

A strong consequence of the proof given for Theorem 1 is the following.

Corollary 3. Let $C, s, \varphi, \eta$, and $\theta(s)$ be as in Corollary 2. Let $R$ be the finite region bounded by $C$. Let $\ell(s, \alpha)$ be a directed line segment that originates at $\varphi(s)$, has an angular direction of $\theta(s)+\alpha$, and has length $\max (0,-\cos (\alpha+\theta(s)-\eta(s-)) / \dot{\theta}(s-)$, $-\cos (\alpha+\theta(s)-\eta(s+)) / \dot{\theta}(s+))$. Then for some constant $\alpha$,

$$
R \subset \bigcup_{s} \ell(s, \alpha) \text {. }
$$

Theorem 1 also has an immediate interpretation as an area minimization formulation.

Corollary 4. Let $P$ be an $n$-gon with side lengths of $\ell_{1}, \ell_{2}, \ldots, \ell_{n}$. Let $\theta_{1}, \theta_{2}, \ldots, \theta_{n}$ be nonnegative values that sum to $2 \pi$. Then

$$
\sum_{j=1}^{n} \frac{1}{4} \ell_{j}^{2} \cot \left(\frac{\theta_{j}}{2}\right) \geq \operatorname{Area}(P),
$$

and equality holds if and only if $P$ is inscribed in a circle and each $\theta_{j}$ equals the radial angle of the arc subtended by a chord of length $\ell_{j}$.

\section{Proofs of Corollaries 1-4}

The proof of Theorem 1 is deferred to Section 3. The main part of Corollary 1 is established first. Let $\overline{v w}$ denote a segment with length $|\overline{v w}|$. 
Corollary 1(a). Let $P$ be an n-gon with consecutive vertices $v_{1}, v_{2}, \ldots, v_{n}$, and let $S$ be a polygon that is inscribed in a circle, and which has edges with the same lengths as those of $F$. Suppose that $S^{\text {rgn }}$ contains the center of its circumscribing circle. Then

$$
\operatorname{Area}(F) \leq \operatorname{Area}(S),
$$

and equality holds if and only if $F$ can be inscribed in a circle.

Proof. The first step is to show that $S$ exists. Create a path of $n-1$ segments that are congruent to the sides of $P$, apart from some side of maximum length $s$. Let the path have its vertices placed along a circle of huge radius, and consider how the path is forced to curl up as the radius is decreased. Evidently, the endpoints of the path would begin at a distance that equals the sum of the segment lengths, when the circle radius is infinite, and decrease continuously as the radius diminishes. The existence of $P$ ensures that $s$ is no more than this initial sum, so the radius can be decreased until the distance between the endpoints equals $s$. Then the path can be closed with the omitted edge. For completeness, it should be noted that the construction would fail if the diameter of the circle were decreased to a value that is smaller than some edge in the chain of $n-1$ segments, since such a segment cannot be a chord of such a circle. However, since all such lengths are bounded by $s$, and since the diameter must be at least $s$ when the termination condition is satisfied, this failure cannot occur.

Let $S$ have the consecutive vertices $s_{1}, s_{2}, \ldots, s_{n}$. Suppose that $S$ has a

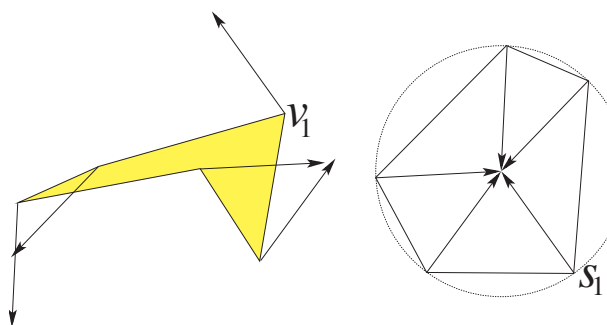

Fig. 3

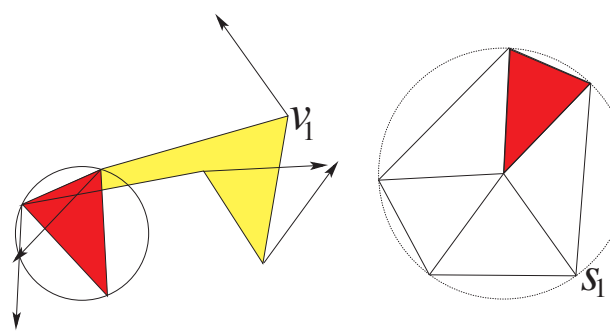

Fig. 4 natural correspondence with $P$ where $\left|\overline{v_{j} v_{j+1}}\right|=\left|\overline{s_{j} s_{j+1}}\right|$ for all $j$. In addition, suppose that $s_{1}, s_{2}, \ldots, s_{n}$ circulate about $S$ with the same rotational sense as $v_{1}, \ldots, v_{n}$ circulate about $P$. Let $c$ be the circumcenter of $S$. Define $\left\{\hat{r}_{j}\right\}_{j=1}^{n}$ to be the monotone slicing of $S$ where $\hat{r}_{j}=\overrightarrow{s_{j} c}$, and let $\left\{r_{j}\right\}_{j=1}^{n}$ be the monotone slicing of $P$ where $r_{j}$ begins at $v_{j}$ and is parallel to $\hat{r}_{j}$. Let the slicing triangles for $\left\{\hat{r}_{j}\right\}_{j=1}^{n}$ be $\hat{t}_{j}=\left(\triangle s_{j+1} s_{j} c\right)^{\mathrm{rgn}}$, for $j=1,2, \ldots, n$.

According to Theorem 1, the rotated slicing rays $\left\{r_{j}(\alpha)\right\}_{j=1}^{n}$ must, for some fixed $\alpha$, induce a set of slicing triangles that cover $P^{\text {rgn }}$. However, the slicing triangle $t_{j}(\alpha)$, if it exists, will have a base that is congruent to the corresponding base of $\hat{t}_{j}$. Moreover, the angle subtending $t_{j}(\alpha)$ 's base must have the same size as $\angle s_{j} c s_{j+1}$ since rotating $r_{j}$ and $r_{j+1}$ by $\alpha$ will keep their angle of intersection the same as that of $\hat{r}_{j}$ and $\hat{r}_{j+1}$, provided $r_{j}(\alpha)$ and $r_{j+1}(\alpha)$ intersect. The Periph- 
eral Angle Theorem in basic Euclidean geometry says that $r_{j} \cap r_{j+1}$ will move along the boundary of a fixed circle and will have, therefore, a maximum altitude precisely when $t_{j}(\alpha)$ is isosceles, in which case it will be congruent to $\hat{t}_{j}$. Of course Area $(S)=$ $\sum_{j=1}^{n} \operatorname{Area}\left(\hat{t}_{j}\right)$, and hence

$$
\operatorname{Area}(P) \leq \sum_{j} \operatorname{Area}\left(t_{j}(\alpha)\right) \leq \sum_{j} \operatorname{Area}\left(\hat{t}_{j}\right)=\operatorname{Area}(S) .
$$

Evidently, equality can only hold if $t_{j}(\alpha) \cong \hat{t}_{j}$ for all $j$, and Area $\left(t_{i} \cap t_{j}\right)=0$, for $i \neq j$, in which case $P$ will be congruent to $S$.

Finally, it should be noted that the edges of $S$ need not have a natural correspondence with those of $P$. Since each $\hat{t}_{j}$ has radial sides of length $r$, where $r$ is the radius of the circumcircle, these triangles can be permuted to ensure that the edges of $S$ are sequenced to correspond with those of $P$.

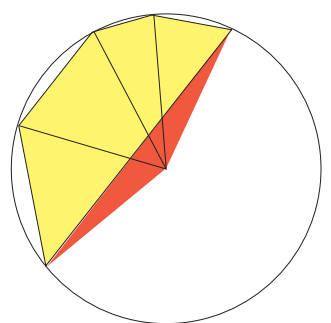

Fig. 5

The more interesting part of Corollary 1, which concerns the case when $S^{\mathrm{rgn}}$ does not contain its circumcenter, is postponed. This case is different because the largest side in $S$ will be the base of a triangle with a reflex angle, and this triangle will contribute a negative term in the formulation of $\operatorname{Area}(S)$ as given in (1). The specifics comprise Corollary 1(b) in Section 4.

Corollary 2 is just a matter of applying Theorem 1 and passing to the limit.

Proof of Corollary 2. Let $C$ be approximated by an $n$-gon $P$. Let the vertices of $P$ be $v_{j}=\varphi(p j / n)$, for $j=1,2, \ldots, n$. Let $r_{j}(\alpha)$ and $r_{j+1}(\alpha)$ be slicing rays with angular directions $\theta(p(j / n))+\alpha$ and $\theta(p((j+1) / n))+\alpha$, and respective origins $v_{j}$ and $v_{j+1}$. Suppose that the two rays intersect, and let their intersection be the point $w_{j}$. Define $\Delta_{j} \equiv\left|\overline{v_{j} v_{j+1}}\right|$, and let $\eta_{j}$ be the angular direction of the outward normal to $\overline{v_{j} v_{j+1}}$. Then by the law of sines, $\left|\overrightarrow{v_{j} w_{j}}\right|=\left(\Delta_{j} / \sin \left(\theta_{j+1}-\theta_{j}\right)\right) \sin \left(\eta_{j}-\pi / 2-\theta_{j+1}-\alpha\right)$, and the area of the slicing triangle is

$$
\operatorname{Area}\left(\Delta v_{j} v_{j+1} w_{j}\right)=\frac{\left(\Delta_{j}\right)^{2}}{2 \sin \left(\theta_{j+1}-\theta_{j}\right)} \cos \left(\eta_{j}-\theta_{j+1}-\alpha\right) \cos \left(\eta_{j}-\theta_{j}-\alpha\right) .
$$

Theorem 1 says that for some $\alpha$,

$$
\sum_{j} \frac{\left(\Delta_{j}\right)^{2}}{2 \sin \left(\theta_{j+1}-\theta_{j}\right)} \cos \left(\eta_{j}-\theta_{j+1}-\alpha\right) \cos \left(\eta_{j}-\theta_{j}-\alpha\right) \geq \operatorname{Area}(P) .
$$

Passing to the limit gives the formulation: for some $\alpha$,

$$
\int \frac{\cos ^{2}(\eta(s)-\theta(s)-\alpha)}{2(d \theta / d s)} d s \geq A .
$$


Proof of Corollary 3 (Sketch). Corollary 3 is little more than an application of Corollary 2 in the limit. The restriction to positive lengths is a consequence of the actual covering argument given for Theorem 2 as stated below, which establishes that every point $x \in P$ will be covered by some slicing triangle $\left(\Delta v_{j} v_{j+1} w_{j}\right)^{\text {rgn }}$ whose base $\overrightarrow{v_{j} v_{j+1}}$ has a counterclockwise orientation with respect to $x$.

Proof of Corollary 4. Similarly, Corollary 4 is just an analytic interpretation of Theorem 1 , since each term $\frac{1}{4} \ell_{j}^{2} \cot \left(\theta_{j} / 2\right)$ is the largest possible area that a triangle with a base length of $l_{j}$ and an opposing vertex angle of $\theta_{j}$ can have, provided $\theta_{j} \leq \pi$.

If some angle $\theta_{j}$ exceeds $\pi$, then the corresponding area term is negative, and additional justification is necessary. For specificity, let the reflex angle be $\theta_{n}$. Let $P$ be an $n$-gon that is inscribed in a circle and has side lengths $\ell_{j}$, for $j=1,2, \ldots, n$, and let its vertices be $v_{1}, v_{2}, \ldots, v_{n}$. Let $Q$ be the $(n+1)$-gon resulting from adjoining $P$ with the exterior isosceles triangle $\Delta v_{0} v_{1} v_{n}$, where vertex $v_{0}$ satisfies $\angle v_{1} v_{0} v_{n}=2 \pi-\theta_{n}$.

Let $r_{1}=\vec{v}_{1} \vec{v}_{0}$, and define $r_{2}, r_{3}, \ldots, r_{n}$ so that $r_{j}$ and $r_{j+1}$ intersect at an angle of $\theta_{j}$. By construction, $r_{n}=\overrightarrow{v_{n} v_{0}}$. Theorem 2 and its mild extension in Corollary 5(a) guarantee that

$$
\sum_{j=1}^{n-1} \frac{1}{4} \ell_{j}^{2} \cot \left(\frac{\theta_{j}}{2}\right) \geq \operatorname{Area}(Q)
$$

It then follows that

$$
\sum_{j=1}^{n-1} \frac{1}{4} \ell_{j}^{2} \cot \left(\frac{\theta_{j}}{2}\right)-\operatorname{Area}\left(\triangle v_{0} v_{1} v_{n}\right) \geq \operatorname{Area}(P) .
$$

Theorem 1 and Corollary 1(b) establish the circumstances where equality can hold.

\section{Proof of Main Theorem}

Theorem 1 follows from the special case described next.

Theorem 2. Let $P$ be an $n$-gon with consecutive vertices $v_{1}, v_{2}, \ldots, v_{n}$ that sequence counterclockwise about $P$, and let $r_{1}, r_{2}, \ldots, r_{n}$ be a monotone slicing for $P$ with associated angles $\theta_{1}, \theta_{2}, \ldots, \theta_{n}$, and associated slicing triangles $\left\{t_{j}\right\}_{j=1}^{n}$. Suppose that $r_{1}=\overrightarrow{v_{1} v_{n}}, r_{n}=\overrightarrow{v_{n} v_{1}}$, and let $v_{1}$ and $v_{n}$ be on the $x$ axis with $v_{1}$ to the right of $v_{n}$, so that $r_{2}, r_{3}, \ldots, r_{n-1}$ point downward. Let $D$ be the upper halfplane. Then

$$
P^{\mathrm{rgn}} \cap D \subset \bigcup_{j=1}^{n-1} t_{j}
$$

Notice that $t_{n}$ is not used to cover $P^{\text {rgn }} \cap D$. This suggests that Theorem 1 might be established by locating a line that partitions $P^{\text {rgn }}$ - convex or otherwise-in a way where Theorem 2 can be applied to each piece. See Fig. 6 . 


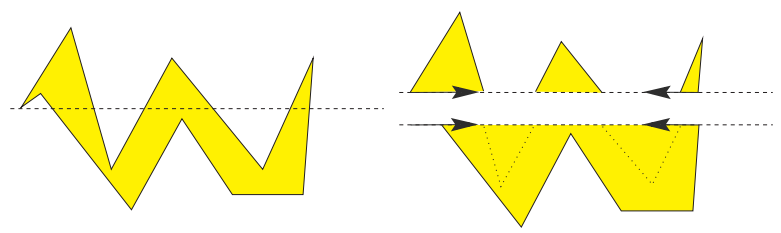

Fig. 6

Proof of Theorem 2. Suppose that $P^{\mathrm{rgn}} \cap D$ is not contained in $\bigcup_{j=1}^{n-1} t_{j}$. Then there is a point $Q \in P^{\mathrm{rgn}} \cap D$ that is in general position and does not belong to $\bigcup_{j=1}^{n-1} t_{j}$. In particular, $Q$ can be selected so that none of the lines from $Q$ through the vertices of $P$ is parallel to any of the rays $r_{1}, r_{2}, \ldots, r_{n}$.

Let the boundary of $P$ be parameterized in polar coordinates with respect to the origin $Q$, and let $(\Theta(t), r(t))$ define the boundary curve, for $t \in[0,1]$. Let $(\Theta(0), r(0))=v_{1}$, and let this parameterization traverse the boundary with a counterclockwise rotation.

Draw a horizontal line $\ell$ through $Q$. By hypothesis, $\ell$ lies above $\overline{v_{1} v_{n}}$. We can assume that the line does not intersect any of the vertices. A polar representation is used to select a subset of edges that all rotate counterclockwise about $Q$, and that define a figure with the following properties. The polygonal figure should intersect $Q$, lie in the closed upper halfplane defined by $\ell$, and be star-convex with respect to $Q$. See the shaded region in Fig. 7. Formally, we find a sequence $t_{0}, t_{1}, \ldots, t_{k}$ where

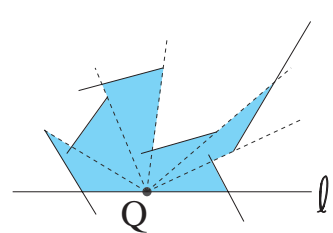

Fig. 7

(a) $0 \leq t_{0}<t_{1}<\cdots<t_{k} \leq 1$,

(b) $\Theta\left(t_{0}\right)<0<\Theta\left(t_{1}\right)<\cdots<\Theta\left(t_{k}\right)<\pi<\Theta\left(t_{k+1}\right)$,

(c) $\Theta\left(t_{i+1}\right)-\Theta\left(t_{i}\right)<\pi$,

(d) $P$ contains an edge that begins at $\left(\Theta\left(t_{j}\right), r\left(t_{j}\right)\right)$, has a counterclockwise orientation with respect to $Q$, and terminates at some location $\left(\Theta\left(w_{j}\right), r\left(w_{j}\right)\right)$ where $\Theta\left(w_{j}\right) \geq \Theta\left(t_{j+1}\right)$, for $j=1,2, \ldots, k$.

The existence of these edges follows from the fact that the boundary has a winding number of 1 about any interior point. An argument that appears to lie within Euclidean geometry is illustrated in Fig. 8, and is sketched as follows.

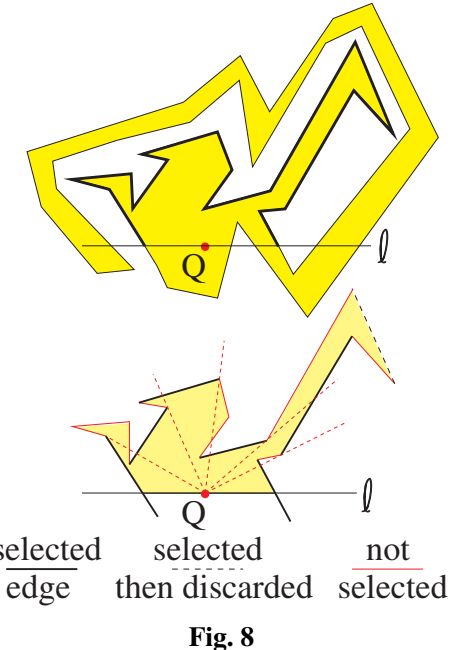

The horizontal line $\ell$ partitions the boundary into a finite number of pieces. Make each piece a closed curve by drawing a line segment between its two endpoints. Of the pieces that are in the upper halfplane, there will be one whose new edge contains $Q$ and is of minimal length. Let this portion of the boundary be called $\varphi$. Let us walk along the edges of $\varphi$ in a counterclockwise direction with respect to the interior of $\varphi$, and monitor the polar angle for the endpoints of each edge. Every time a new maximum is reached in the polar angle, the current edge is selected regardless of whether it can be seen from $Q$. This selected subset is then postprocessed by discarding any edge that cannot be seen from $Q$, because of other edges in the selected subset. 
A more precise formulation is the following. Let $e_{1}, e_{2}, \ldots, e_{z}$ be the edges of $\varphi$ in consecutive order, beginning with the edge that intersects $\ell$ to the right of $Q$. A suitable set of edges can be selected for subsequent postprocessing as follows:

Create the empty set $S$;

$\Theta \leftarrow 0$;

for $j \leftarrow 1$ to $z$ do

Assign the maximum of the polar angle values for the endpoints of $e_{j}$ to $\alpha$;

if $\alpha>\Theta$ then $\quad\{\alpha$ is a new maximum angle $\}$

$\Theta \leftarrow \alpha ; \quad\{$ Remember the new maximum $\}$

Include $e_{j}$ in $S$

endif

endfor.

The postprocessing is equally direct; the interested reader may wish to consider how to design an elegant solution.

Let each of the resulting edges (after postprocessing) be

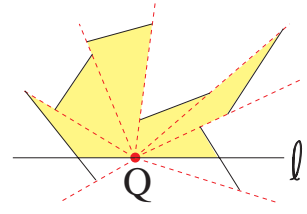

Fig. 9 trimmed so that consecutive segments have an endpoint on a common radial line as shown. Let the first and last segment in the sequence penetrate below $\ell$ as illustrated in Fig. 9. Let $s_{1}, s_{2}, \ldots, s_{k}$ be the sequence of segments. The desired set of angles $\left\{\Theta\left(t_{i}\right)\right\}$ is defined by the angles of the radial lines through the endpoints of these segments.

Each $s_{i}$, for $i=1,2, \ldots, k$, will be assigned two slicing rays. Let $s_{i}$ be a trimmed version of the edge $e_{j}$, then each endpoint of $s_{i}$ will have an emanating slicing ray that is parallel to the ray from the corresponding endpoint of $e_{j}$.

Since $s_{i}$ has rays that are parallel to the rays of its corresponding untrimmed edge, the slicing triangle formed from any $s_{i}$ and its rays must be contained within the corresponding slicing triangle for $P$. Thus, it suffices to show that $Q$ is contained in the slicing triangle formed from some $s_{i}$ and its associated rays.

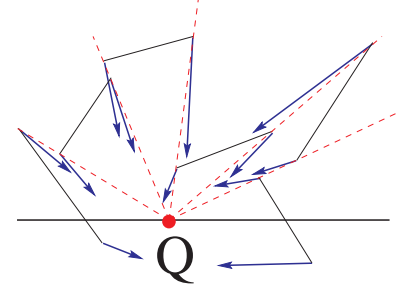

Fig. 10

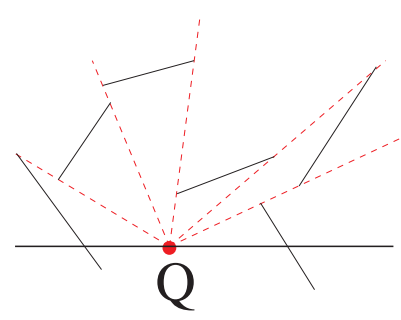

Fig. 11
Since none of the original slicing rays was parallel to any of the lines from $Q$ through the vertices of $P$, none of the slicing rays associated with the $s_{i}$ will pass through $Q$. Let a slicing ray be called right-oriented if it begins above $\ell$ and intersects $\ell$ to the right of $Q$. Similarly, a ray is left-oriented if it begins above $\ell$ and intersects $\ell$ to the left of $Q$.

For any edge $s_{i}$, let its two endpoints be denoted by the forward endpoint and the rear endpoint, where the polar angle for the forward endpoint has a larger value than that of the rear. Similarly, each radial line will have two incident edges, which are identified as forward and rear. As shown in Fig. 11, the edges $s_{1}, s_{2}, \ldots, s_{k}$ have a natural order that begins with an edge that intersects $\ell$ on the right, and proceeds in counterclockwise order through to the last edge, which intersects $\ell$ on the left. 


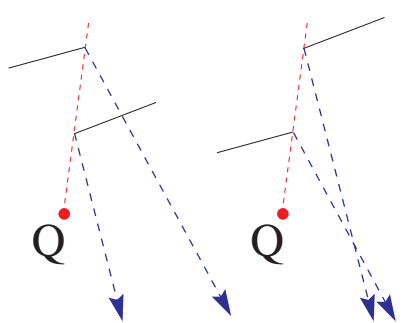

Fig. 12

Remark: as Fig. 12 shows, if the ray emanating from the forward endpoint of a radial line's rear incident edge is right-oriented, then so is the ray that emanates from the rear vertex of the radial line's forward edge. This property is just a straightforward consequence of the monotone rotational increment in the direction of consecutive slicing rays. Of course, the analogous fact holds when the words left and right are exchanged, along with the notions forward and rear.

To locate a slicing triangle that covers $Q$, let these edges be searched in sequential polar order for the first associated ray that is left-oriented. In view of the preceding remark, this ray, if it exists, must emanate from the forward endpoint of its associated segment. There are three cases.

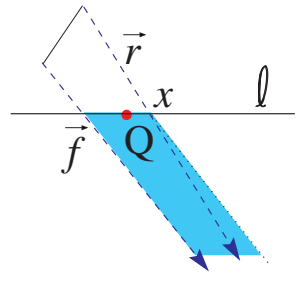

Fig. 13

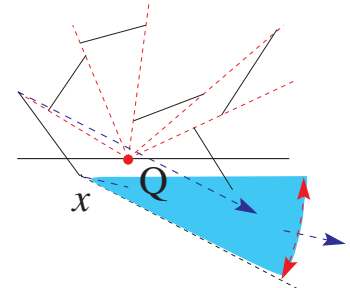

Fig. 14

Case 1. The sought ray is associated with a segment that lies entirely above $\ell$. Let the segment's rear ray be $\vec{r}$, and let the forward ray be $\vec{f}$. In this situation, $Q$ is trapped between $\vec{f}$ and $\vec{r}$. Let $\vec{r}$ intersect $\ell$ at $x$. Draw a ray at $x$ that is parallel to $\vec{f}$. These two rays define a half infinite strip that $\vec{r}$ must enter at $x$ (due to monotonicity). Hence, $\vec{f}$ and $\vec{r}$ must intersect below $\ell$ to form a slicing triangle that contains $Q$.

Case 2. All segments that lie entirely above $\ell$ have emanating rays that are right-oriented. In this circumstance, the reasoning of Case 1 applies to segment $s_{k}$, which has a forward endpoint $x$ that lies below $\ell$. Notice that the associated ray emanating from $x$ must lie in the cone that has its vertex at $x$, one side parallel to the ray associated with the rear of $s_{k}$ (due to monotonicity) and the other parallel to $\ell$ and pointing to the right (since no ray can be rising).

Case 3. All segments that lie entirely above $\ell$ have emanating rays that are leftoriented. This circumstance is, mutatis mutandis, the same as Case 2 with all notions of left and right exchanged.

To ensure correctness, we are obliged to discuss a case that cannot occur. The underlying question is why the above argument fails to hold for the portion of the polygon that lies below the $x$-axis. The answer is that such points $Q$ will not be trapped by either $r_{1}$ or $r_{n}$, and therefore might not be caught between any pair of intermediate rays. Moreover, the monotonicity argument as illustrated in Fig. 12 would not hold if $\overline{v_{n} v_{1}}$ were an intermediate (and omitted) edge that belonged to the portion of boundary named $\varphi$. However, this event cannot occur since $\varphi$ lies above $\ell$.

The proof is now complete.

Two mild extensions of Theorem 2 are needed to complete the proof of Theorem 1.

Corollary 5. Let $P$ be an $n$-gon with consecutive vertices $v_{1}, v_{2}, \ldots, v_{n}$, and let $r_{1}, r_{2}, \ldots, r_{n}$ be a monotone slicing for $P$ with associated angles $\theta_{1}, \theta_{2}, \ldots, \theta_{n}$, and 
associated slicing triangles $\left\{t_{j}\right\}_{j=1}^{n}$. Let $v_{1}$ and $v_{n}$ be on the $x$-axis, with $v_{1}$ to the right of $v_{n}$. Let $D$ be the upper halfplane.

(a) Suppose that $r_{1}$ and $r_{n}$ intersect, and the intersection point lies below the $x$-axis. Then

$$
P^{\mathrm{rgn}} \cap D \subset \bigcup_{j=1}^{n-1} t_{j} .
$$

(b) Suppose that $r_{1}$ points at $v_{n}, v_{n-1}$ lies in the upper halfplane, and $r_{n-1}$ intersects the $x$-axis at or to the left of $v_{n}$. Then

$$
P^{\mathrm{rgn}} \cap D \subset \bigcup_{j=1}^{n-1} t_{j} .
$$

Proof. For both (a) and (b), the rays $r_{2}, \ldots, r_{n-1}$ are constrained to point downward. Moreover, if $r_{1}$ and $r_{n}$ are replaced by rays that point at at each other, then the slicing rays would still be monotone and satisfy the conditions of Theorem 2 .

For (a), this change only affects three slicing triangles, which each might lose coverage below the $x$-axis but are otherwise unaffected.

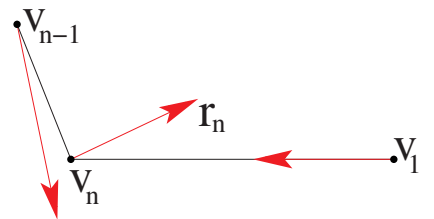

Fig. 15

For (b), only triangles $t_{n-1}$ and $t_{n}$ could possibly be affected by the change in $r_{n}$. However, $t_{n}$ will remain fixed (as a segment with zero area), and $t_{n-1}$ will not exist for either direction of $r_{n}$, due to the errant pointing of $r_{n-1}$, unless it points at $v_{n}$, in which case $t_{n}$ will also be a degenerate triangle. So the coverage will be the same.

The intended application for part (b) is instances where $r_{n}$ points upward as shown.

Proof of Theorem 1. Let, for $j=1, \ldots, n$, angle $\beta_{j}$ satisfy $\beta_{j}=\theta_{j+1}-\theta_{j}$. Intuitively, $\beta_{j}$ is associated with edge $\overline{v_{j} v_{j+1}}$. Suppose, for the moment, that $\beta_{j}<\pi$, for all $j$.

Given an edge and its associated angle $\beta$, a suitable interpolation scheme is needed to compute the appropriate portion of $\beta$ to associate with a given portion of the edge. The interpolation, it turns out, will only be needed for edges that are intersected by a transversal.

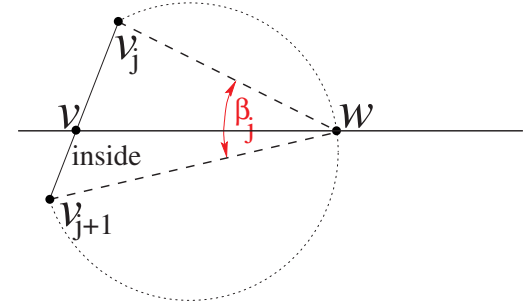

Fig. 16
Let the transversal $\ell$ intersect the edge $\overline{v_{j} v_{j+1}}$ at the point $v$, and suppose that one side of $\overline{v_{j} v_{j+1}}$ is designated as the inside. Let $w$ be a point on $\ell$ that is on the inside half of $\ell$ as defined by the designation for $\overline{v_{j} v_{j+1}}$, and suppose that $\angle v_{j} w v_{j+1}=\beta_{j}$. Then the interpolation rule associates $\angle v w v_{j+1}$ with the segment $\overline{v v_{j+1}}$, and $\angle v_{j} w v$ with the segment $\overline{v_{j} v}$.

The interpolation procedure introduces $v$ as an artificial vertex on $\overline{v_{j} v_{j+1}}$. The vertex will also need an associated slicing ray $r_{v}$. Let $r_{v}$ emanate from $v$ with an angular direction of $\theta_{v} \equiv \theta_{j}+\angle v_{j} w v$. 
Now let $\ell$ be a horizontal line that lies above $P$, and let $\ell$ be translated downward in a continuous motion, so that $\ell$ will eventually intersect $P$ and will eventually wind up below the polygon. We can suppose that $\ell$ is not parallel to any of the lines defined by pairs of vertices of $P$. Let this family of sweep lines be denoted by $\ell(\rho)$, where $\rho$ ranges from 0 to 1 .

Let $p_{1}(\rho)$ be the rightmost intersection of $\ell(\rho)$ with the boundary of $P$, and let $p_{2}(\rho)$ be the leftmost such point. For transversals of interest, $p_{1}(\rho)$ and $p_{2}(\rho)$ will split the boundary of $P$ into two pieces, which we name the upper and lower boundaries.

Let $\sigma_{1}(\rho)$ and $\sigma_{2}(\rho)$ be the sums of the angles associated with the segments belonging to, respectively, the upper and lower boundaries of $P$. If $p_{1}(\rho)$ or $p_{2}(\rho)$ are not vertices, the interpolation rule is used to apportion a fraction of the relevant angle(s) to each sum.

Ideally, there ought to be a $\rho$ and transversal $\ell(\rho)$ where $\sigma_{1}(\rho)=\sigma_{2}(\rho)=\pi$. Although this would would be evident if $\sigma(\rho)$ were continuous, the function can have jumps when $\ell$ intersects a vertex. We defer, momentarily, an analysis of the discontinuous case, which includes the possibility that the interpolation procedure might fail.

So suppose that there is a $\rho_{0}$ where $\sigma_{1}\left(\rho_{0}\right)=\sigma_{2}\left(\rho_{0}\right)=\pi$. Then the conditions of Theorem 2 can be satisfied as follows. First, interpolation is used to create artificial vertices and associated rays as needed at $p_{1}\left(\rho_{0}\right)$ and $p_{2}\left(\rho_{0}\right)$. Let $\alpha$ be the angle between the ray emanating from $p_{1}\left(\rho_{0}\right)$ and a ray pointing from $p_{1}\left(\rho_{0}\right)$ to $p_{2}\left(\rho_{0}\right)$. Then a rotation of the slicing rays by $\alpha$ will force the ray emanating from $p_{1}\left(\rho_{0}\right)$ to point at $p_{2}\left(\rho_{0}\right)$. Similarly, the ray emanating from $p_{2}$ will point at $p_{1}\left(\rho_{0}\right)$.

In this case, Theorem 2 shows that the coverage is complete for both halves of $P^{\text {rgn }}$ as determined by $\ell\left(\rho_{0}\right)$. Moreover, the interpolation rule ensures that the artificial rays are unnecessary, since any such ray and its two neighboring rays intersect at a common point as illustrated in Fig. 16.

However, this procedure process might fail for three reasons.

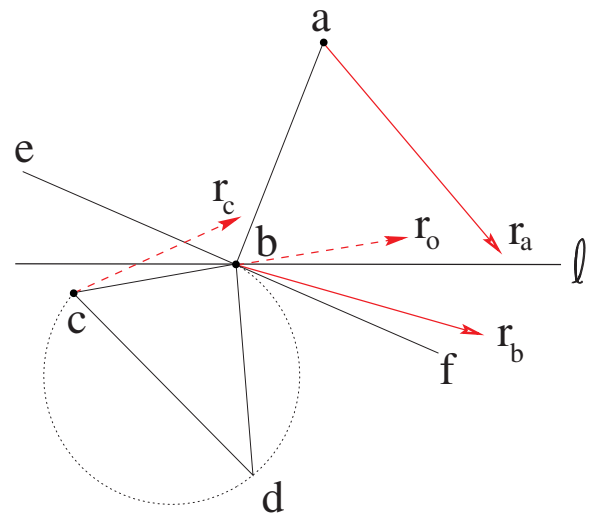

Fig. 17
First, there is a discontinuity that can occur from a failure in the interpolation rule. As shown in Fig. 17, the transversal is horizontal. The point $b$ is the current $p_{2}$, so that $\ell$ does not intersect $P$ to the left of $b$. The rotational value $\beta$ associated with edge $\overline{b c}$ is $\angle b d c$. The possible locations for vertex $d$ as used in the interpolation procedure is shown as the circular arc $b \widehat{d c}$. Line $\overline{e f}$ is tangent to $b \widehat{d c}$ at $b$.

Suppose that the rays have been rotated so that the ray at $p_{1}$, which is not shown, points at $p_{2}$. In this case, the ray $r_{b}$, which emanates from $p_{2}$, has an associated angle $\theta_{b}=\sigma_{2}+\pi$ that is a little less than the necessary $2 \pi$. The ray $r_{c}$ is the slicing ray for vertex $c$, and has an associated angle equal to $\theta_{b}+\angle b d c$.

Because $r_{b}$ points downward from $p_{2}$ (and the ideal direction is to point at $p_{1}$ ), the descent of $\ell$ should, in principle, be continued. Such a change would cause additional 
rotation to occur from the relocation of both $p_{1}$ and $p_{2}$. The difficulty is that in the illustration, the interpolated contribution from $\overline{b p_{2}(\rho)}$, once $\ell(\rho)$ partitions $\overline{b c}$, has a discontinuous jump.

It is easy to see that the interpolation rule will be jump-free at a vertex $b$ if and only if the line that is parallel to $\ell$ and contains $b$ also intersects the interior of $\operatorname{arc} b \widehat{d c}$ or is tangent at $b$.

However, when this criterion fails, descending $\ell$ from $b$ introduces a jump $\theta_{J}$ in the interpolated angle, which equals the angle between the tangent $\overrightarrow{b f}$ and the rightward pointing transversal from $b .^{2}$ To resolve this case, it suffices to use the transversal through $b$. The upper boundary will have monotone slicing rays that begin with one pointing from $p_{1}$ toward $p_{2}$, and end with $r_{b}$, which has some kind of downward direction. Corollary 5(a) addresses this case.

As for the lower half, Corollary 5(b) will apply if $r_{c}$, which is the penultimate slicing ray for the lower half of $P$ (as viewed from a mirrored perspective for Corollary 5), intersects $\ell$ at or to the left of $p_{2}$ as illustrated. To prove that $r_{c}$ satisfies this condition, let $r_{0}$ be collinear with $\overline{b c}$ and point upward from $b$. Then the angle between $r_{0}$ and the tangent $\overrightarrow{b f}$ is precisely $\angle b d c$. Failure will occur only if $r_{b}$ lies within the cone bounded by $\overrightarrow{b f}$ and $r_{0}$, since $r_{b}$ must point downward, and the smallest interpolated angle that can be constructed from $\angle b d c$ must be too large. Consequently, a rotation of $r_{b}$ by $\theta_{J}$ will cause it to become rising, and a rotation by $\angle b d c$ will cause it to pass $r_{0}$. However, the rotation by $\angle b d c$ gives a ray emanating from $b$ that is parallel to $r_{c}$. Consequently, $r_{c}$ has a direction that comprises a positive rotation of $\overrightarrow{c b}$ with respect to $c$, and a rotation of the falling $r_{b}$ by an angle equal to $\angle b d c<\pi$. It follows that $r_{c}$ rises to intersect $\ell$ at or to the left of $b$, which ensures that Corollary 5(b) is applicable.

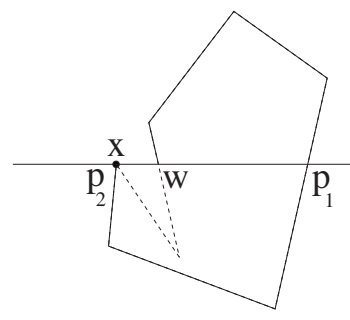

Fig. 18

Second, suppose that $\ell(\rho)$ intersects a vertex $x$ that has both of its connecting edges on the same side of the transversal, and suppose that $x$ equals $p_{1}(\rho)$ or $p_{2}(\rho)$. For specificity, let $x=p_{2}(\rho)$, and let the edges connecting $p_{2}(\rho)$ lie in the lower halfplane bounded by $\ell(\rho)$. Let $w$ be, in this case, the next-to-the-leftmost intersection of $\ell(\rho)$ and the boundary of $P$ (i.e., the leftmost intersection that is to the right of $\left.p_{2}(\rho)\right)$. By the choice of $\ell, w$ cannot be a vertex of $P$ and must therefore belong to some edge that crosses $\ell$.

In this circumstance, it is possible that $\sigma_{2}(\rho)$ might jump from a value greater than $\pi+\varepsilon$ to a value that is less than $\pi-\varepsilon$ as the transversal descends to $x$. This jump is harmless, since the covering problem for the upper and lower halves of $P$ can be treated separately. An artificial vertex at $p_{1}(\rho)$ is added to both boundary portions, and the upper boundary is terminated at the artificial vertex $w$. The interpolation rule determines the angular portions to assign each subsegment of a partitioned edge. Lastly, a slicing ray is

\footnotetext{
${ }^{2}$ Notice that $c$ must lie below $\ell$ as otherwise the discontinuity must be of the first type associated with Fig. 18. Because $c$ lies below $\ell$, segment $\overline{b c}$ induces no comparable jump at $c$. Similarly, the segment would not induce a jump at $b$ if $\overrightarrow{b c}$ were to point down and to the right, rather than down and to the left, because the upper side of $\overline{b c}$ would then be designated as the inside.
} 
introduced at $p_{1}(\rho)$, along with an angular adjustment $\alpha$ that forces the ray at $p_{1}(\rho)$ to point toward $p_{2}(\rho)$. Vertex $w$ also gets a slicing ray.

Since neither collection of edges will have a total rotational sum that exceeds $\pi$, Corollary 5(a) suffices to establish the coverage of each half.

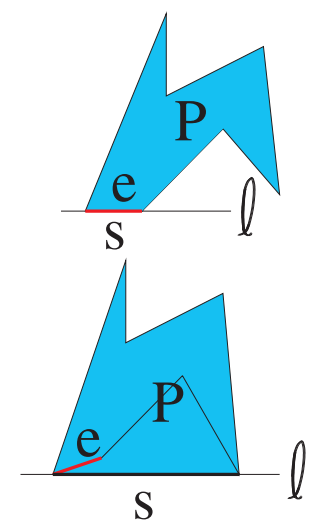

Fig. 19

Lastly, suppose that some edge $e$ has an associated angle of size $\pi$ or more. We show that such an occurrence is harmless; in fact, $e$ 's slicing triangle is not needed to cover $P^{\text {rgn }}$.

Let $s$ be a longest segment that belongs to the convex hull of $P$ and either contains $e$ or seals off $e$ inside of the region enclosed by $P \cup s$. Let $P$ be redefined to include the enclosed pocket, and let the angle associated with $s$ equal the sum of the angles associated with the edges that no longer belong to $P$, including $e$. Thus, the associated angle is at least $\pi$. Let the extension of $s$ be the transversal $\ell$, so that the endpoints of $s$ are $p_{1}$ and $p_{2}$.

Let the rays be rotated so that the ray from $p_{1}$ points at $p_{2}$. Then each slicing ray will intersect $\ell$, and Corollary 5(a) shows that the coverage is complete.

\section{Minor Extensions}

For completeness, we now give a reduction to show that Corollary 1(b) follows from Corollary 1(a). A direct proof of Corollary 1(b) is given at the conclusion of this section.

Corollary 1(b). Let $F$ be a polygon, and let $S$ be a polygon that is inscribed in a circle, and which has edges with the same lengths as those of $F$. Suppose that $S^{\mathrm{rgn}}$ does not contain the center of its circumscribing circle. Then the area of $S$ is at least as large as that of $F$.
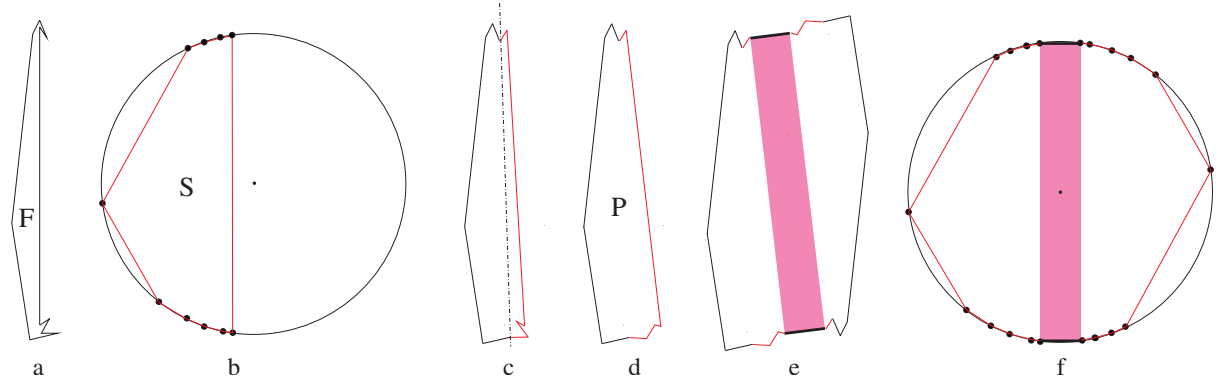

Fig. 20

Proof (Sketch). A first step is to use reflection and other simple local improvement transformations to expose the longest edge so that its extension as an infinite line defines 
a halfplane that contains the modified polygon. Let the new region be $P$. Then $P$ and a rotated copy of $P$ can be combined with a (virtual) rectangular filler to build a new figure that will have the same size circumscribing circle as shown, and that meets the conditions of Corollary 1(a). If $P$ has $n$ edges, then the composite figures will have $2 n$ edges. In particular, each edge in $P$, apart from the largest, will have two comparable edges in the composite. The last two edges in the composite will represent the top and bottom edges of the filler rectangle. By construction, the filler rectangle in the modified polygon and the rectangle inscribed in the circle have a pair of sides congruent to the longest segment in $P$, and top and bottom segments that span twice the distance from the longest edge of the inscribed polygon (in Fig. 20(b)) to the center of its circumcircle.

Lastly, we end this section by outlining extensions of Theorem 2 to cases where the rays $r_{1}$ and $r_{n}$ do not point at each other. Although Corollary 5 addressed this case, it was somewhat weak.

Theorem 3. Let $P$ be an $n$-gon with consecutive vertices $v_{1}, v_{2}, \ldots, v_{n}$ that sequence counterclockwise about $P$, and let $r_{1}, r_{2}, \ldots, r_{n}$ be a monotone slicing for $P$ with associated angles $\theta_{1}, \theta_{2}, \ldots, \theta_{n}$, and associated slicing triangles $\left\{t_{j}\right\}_{j=1}^{n}$. Let $v_{1}$ and $v_{n}$ be on the $x$-axis with $v_{1}$ to the right of $v_{n}$, and suppose that $r_{1}$ and $r_{n}$ intersect at a unique point $p_{1}$.

Define $\hat{r}_{1}$ to be the ray that emanates from $p_{1}$ and is parallel to (and coincides with) $r_{1}$. Let $\hat{r}_{n}$ be the ray that emanates from $p_{1}$ and is parallel to (and coincides with) $r_{n}$. Let $C$ be the cone defined by the boundary $\hat{r}_{1} \cup \hat{r}_{n}$.

(a) If $p_{1}$ lies above the line $\overleftrightarrow{v_{1} v_{n}}$, then

$$
P^{\mathrm{rgn}} \cap C \subset \bigcup_{j=1}^{n-1} t_{j}
$$

(b) If $p_{1}$ lies below $\overleftrightarrow{v_{1} v_{n}}$, then

$$
\left(P^{\mathrm{rgn}} \backslash C\right) \cup t_{n} \subset \bigcup_{j=1}^{n-1} t_{j}
$$

Proof (Sketch). The basic reasoning is the same as that of Theorem 2. The only difference is that the horizontal line $\ell$ through $Q$ is replaced by a pair of rays that emanate from $Q$ and are antiparallel to $\hat{r}_{1}$ and $\hat{r}_{n}$ as shown.

For completeness, we observe that Theorem 3(b) facilitates a direct proof of Corollary 1(b) without any need to transform the problem into an instance of Corollary 1(a).

Direct Proof of Corollary 1(b). Let $F$ be oriented. If the figure is not convex, let $\ell=$ $\overleftrightarrow{v_{i} v_{j}}$ be a global support line that intersects $F$ at $v_{i}$ and $v_{j}$, for $i \neq j$. 

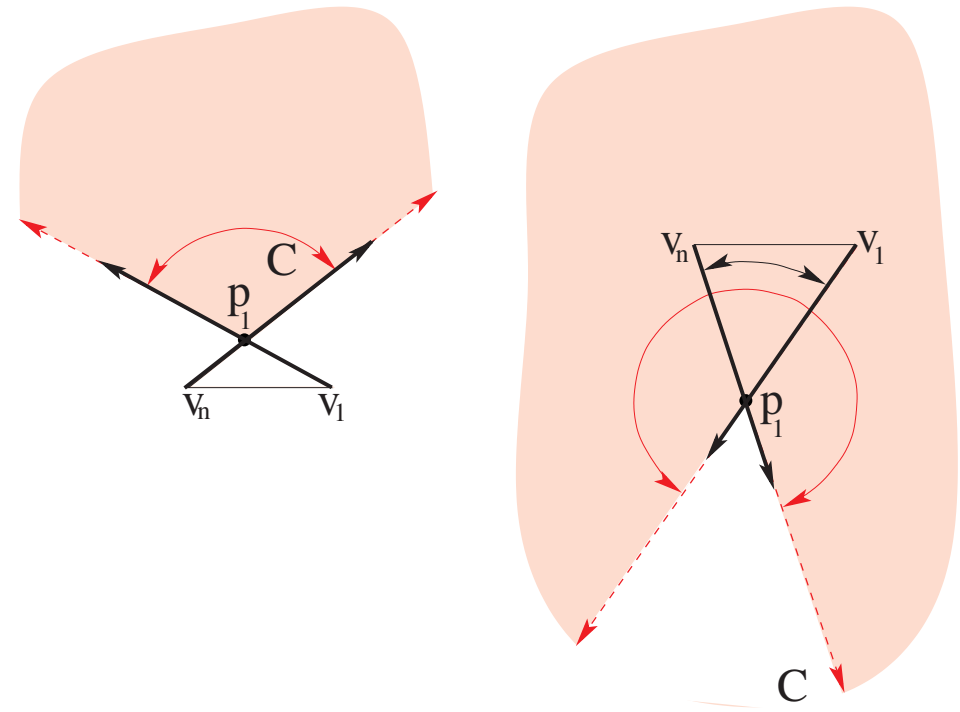

Fig. 21
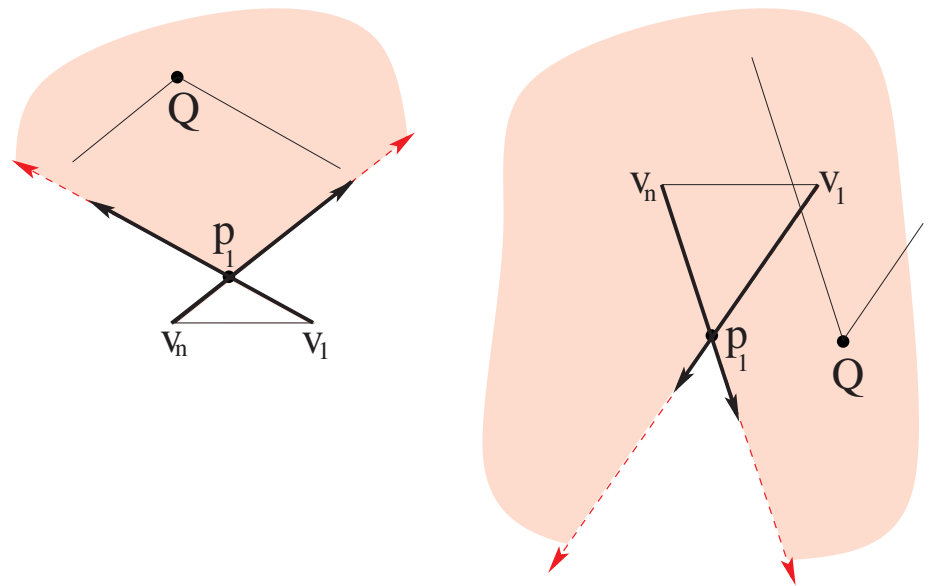

Fig. 22

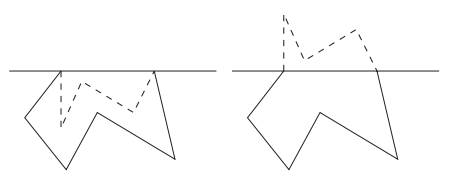

Fig. 23

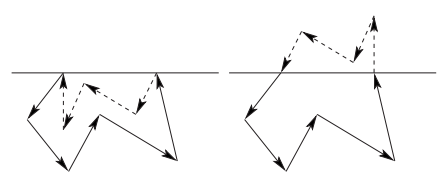

Fig. 24

Suppose that $F \cap \overline{v_{i} v_{j}}=\left\{v_{i}, v_{j}\right\}$, so that $\overline{v_{i} v_{i}}$ seals off some pocket of $F$ as shown. Steiner symmetrization flips (reflects) one of the subpaths terminating at $v_{i}$ and $v_{j}$ about $\ell$ to increase the area of the resulting figure while preserving the edge lengths.

As a practical matter, it is simpler to reverse the sequencing of these edges, which effectively rotates the boundary portion about the midpoint of $\overline{v_{i} v_{j}}$ (but does not reverse their direction). While iterations of either operation lead, eventually, to a convex region 
with increased area, the virtual rotation operation is easier to analyze. It simply rearranges the segment ordering without changing their directions. Since this procedure can produce no more than $(n-1)$ ! such arrangements, one of these polygons must have a maximal area. This polygon—call it $R$-must be convex, since otherwise this procedure could further increase the area.

At this point, the mapping between $S^{\mathrm{rgn}}$ and the convex $R$ is straightforward. Let radial slices from the vertices of $S$ to the center of its circumcircle form the triangular regions $h_{1}, h_{2}, \ldots, h_{n}$, with $h_{n}$ exterior to $S$, so that $S^{\mathrm{rgn}}=\left(\bigcup_{i=1}^{n-1}\right) \backslash h_{n}$. The radial rays of $S$ should be transferred to $R$ with a rotational offset that forms $t_{n} \cong h_{n}$ as an isosceles triangle exterior to $R^{\text {rgn }}$. It follows that

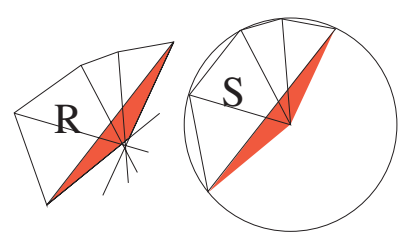

Fig. 25

$$
\begin{aligned}
\operatorname{Area}(R) & \leq \sum_{j=1}^{n-1} \operatorname{Area}\left(t_{j}\right)-\operatorname{Area}\left(t_{n}\right) \\
& \leq \sum_{j=1}^{n-1} \operatorname{Area}\left(h_{j}\right)-\operatorname{Area}\left(h_{n}\right)=\operatorname{Area}(S),
\end{aligned}
$$

and equality holds iff $R$ can be inscribed in a circle.

This construction resolves a question implicitly posed by Yaglom and Boltanskiĭ about the prospects of finding a geometric proof of Corollary 1 that does not rely on an intermediate isoperimetric inequality [8, p. 56].

\section{A Geometric Construction Algorithm}

The proof of Theorem 1 gives an algorithmic construction in traditional Euclidean geometric algebra.

Let $P$ be an $n$-gon, and let $Q$ be a polygon that is inscribed in a circle and has sides that are congruent to those of $P$. Let the vertices of $P$ be $v_{1}, v_{2}, \ldots, v_{n}$, and be in correspondence with $q_{1}, q_{2}, \ldots, q_{n}$ in $Q$. Let both vertex sets have the same orientation about their respective interiors. Let $q_{0}$ be the center of the circle circumscribing $Q$. Suppose that $v_{1}$ is a corner of the convex hull of $P$.

Form the slicing triangles $\hat{t}_{j}=\left(\Delta q_{j} q_{0} q_{j+1}\right)^{\mathrm{rgn}}$, for $j=1,2, \ldots, n$. Draw a line through the points $q_{1}$ and $q_{0}$. Let the line exit $Q^{\text {rgn }}$ at $q \in \overline{q_{k} q_{k+1}}$ for some vertex index $k$. The proof of Theorem 1 shows that a suitable transversal $\ell$ is either $\overrightarrow{v_{1} v_{k}}, \overrightarrow{v_{1} v_{k+1}}$, or $\overrightarrow{v_{1} v}$, where $v$, if it exists, is located so that $\angle v_{1} v v_{k}=\angle q_{1} q q_{k}, \angle v_{k} v v_{k+1}=\angle q_{k} q q_{k+1}$, and $v \in\left(\triangle v_{1} v_{k} v_{k+1}\right)^{\mathrm{rgn}}$.

In any case, the image of $\hat{t}_{j}$ is defined by $\overline{v_{j} v_{j+1}}$ and the intersection (if it exists) of the slicing rays $r_{j}$ and $r_{j+1}$. Ray $r_{1}$ emanates from $v_{1}$ with an angular direction $\theta_{1}$ that points along $\ell$. Ray $r_{j}$ emanates from $v_{j}$ and has the direction $\theta_{j}=\theta_{j-1}+\angle q_{j-1} q_{0} q_{j}$, for $j=2,3, \ldots, n$.

Evidently, the construction is trivial, but its proof of correctness is somewhat subtle. On the other hand, each step of this topological approach is elementary. Yet the perspective gives a variety of similar results that are sometimes not so obvious. 
This paper grew out of an effort to find a correct elementary proof of the basic isoperimetric inequality in two dimensions. That effort was successful, but a web-based search showed that Lawlor had discovered the approach about two years earlier [3]. Other developments based on divide-and-conquer and triangular decompositions can be found in [5]-[7]; related questions appear in [1], [2], and [4].

\section{Acknowledgments}

It is a pleasure to thank H.H. Wu and J. Pach for stimulating discussion and an unerring sense of inquiry.

\section{References}

1. Bezdek, A., and K. Bezdek. On a discrete Dido-type question. Elem. Math., 4 (1989), 92-100.

2. Fejes Tóth, L. Über das Didosche Problem. Elem. Math., 23 (1968), 97-101.

3. Lawlor, G. A new area maximization proof for the circle. Math. Intelligencer, 20 (1999), 29-30.

4. Osserman, R. The isoperimetric inequality. Bull. Amer. Math. Soc., 84 (1978), 1182-1238.

5. Siegel, A. Some Dido-type inequalities. Elem. Math., 56 (2001), 1-4.

6. Siegel, A. A Dido problem as modernized by Fejes Tóth. J. Discrete Comput. Geom., 27(2) (2002), 227-38.

7. Siegel, A. An isoperimetric inequality for self-intersecting polygons. Submitted for publication.

8. Yaglom, I.M., and V.G. Boltanskir. Convex Figures (translated by P.J. Kelly and L.F. Walton). Holt, Rinehart and Winton, New York, 1961.

Received April 16, 2001, and in revised form July 25, 2002. Online publication December 20, 2002. 